Editor's Note: These short reviews of recent JNeurosci articles, written exclusively by students or postdoctoral fellows, summarize the important findings of the paper and provide additional insight and commentary. If the authors of the highlighted article have written a response to the Journal Club, the response can be found by viewing the Journal Club at www.jneurosci.org. For more information on the format, review process, and purpose of Journal Club articles, please see http://jneurosci.org/content/ preparing-manuscript\#journalclub.

\title{
Electrical Stimulation of the Pulvinar Disrupts Control of Spatially Directed Actions
}

\author{
Naomi N. Odean ${ }^{1,2}$ and Natalie A. Steinemann ${ }^{1}$ \\ ${ }^{1}$ Zuckerman Mind Brain Behavior Institute, Howard Hughes Medical Institute, Department of Neuroscience, Columbia University, New York, New York \\ 10027, and ${ }^{2}$ Doctoral Program in Neurobiology and Behavior, Columbia University, New York, New York 10032 \\ Review of Dominguez-Vargas et al.
}

The dorsal pulvinar is a thalamic nucleus that remains poorly understood despite being the subject of many studies. Inputs from the superior colliculus (Benevento and Standage, 1983) and reciprocal connections with parietal (Hardy and Lynch, 1992; Gutierrez et al., 2000) and prefrontal (Ma et al., 1998; Gutierrez et al., 2000) cortices provide the pulvinar with the anatomical connectivity needed to influence visually guided behavior. Dorsal pulvinar neurons have large contralateral visual receptive fields and show saccade-related activation (Petersen et al., 1985). A role in visual attention and modulation of cortical activity has also been suggested for dorsal pulvinar (Olshausen et al., 1993; Buschman and Kastner, 2015), but the complex response patterns of pulvinar neurons have made it difficult to confirm their function and network dynamics.

Perturbation of neural activity is a useful tool for exploring the functions of various brain regions, including the pulvinar. Indeed, inactivation studies have provided some insights into pulvinar func-

Received April 21, 2017; revised June 7, 2017; accepted June 8, 2017.

We thank Michael Shadlen, Sean Escola, Mehdi Sanayei, Christopher Cueva, and Igor Kagan for comments on the manuscript.

The authors declare no competing financial interests.

Correspondence should be addressed to Dr. Natalie A. Steinemann, 630

West 168th Street, P\&S Building Room 16-409 (MailBox 102-A), New York, NY 10032.E-mail:ns3058@columbia.edu.

DOI:10.1523/JNEUROSCI.1079-17.2017

Copyright $\odot 2017$ the authors $\quad 0270-6474 / 17 / 376811-03 \$ 15.00 / 0$ tion, indicating that the nucleus might be involved in identifying action-relevant stimuli, allocating or maintaining spatial attention, and initiating directed movements. Unilateral inactivation of the dorsal pulvinar results in spatial neglect, contralesional saccadic slowing, a bias toward choosing ipsilesional targets, and impaired saccadic and manual motor control (Petersen et al., 1987; Wilke et al., 2010, 2013), whereas injections of the GABA receptor antagonist bicuculline result in saccadic response time effects consistent with enhanced attentional orientation toward the contralateral visual field (Petersen et al., 1987). However, these studies are limited by the timescale of pharmacological perturbation. More nuanced conclusions might be drawn using temporally and spatially more selective techniques, such as electrical stimulation or optogenetic approaches.

To gain such insight into pulvinar function, Dominguez-Vargas et al. (2017) applied unilateral electrical stimulation to the dorsal pulvinar of two macaques while they performed fixation and saccade tasks. During the fixation task, stimulation applied $500 \mathrm{~ms}$ after fixation was acquired, evoked short eye movements toward the contralateral visual field in one monkey at high stimulation currents.

Dominguez-Vargas et al. (2017) next applied stimulation during a visually guided saccade task in which monkeys were shown one or two targets and were rewarded for making a saccade to either target. Stimulation was applied at seven $200 \mathrm{~ms}$ time windows between $120 \mathrm{~ms}$ before and $120 \mathrm{~ms}$ after cue onset. In this task, early electrical stimulation, which overlapped with the fixation period, decreased reaction times toward ipsilateral targets and created an ipsilateral choice bias. Notably, stimulation during fixation increased ipsilateral responses in the saccade task but evoked contralateral responses in the fixation task. However, electrical stimulation commencing after the appearance of visual targets generated a contralateral bias and delayed the onset of saccades into both hemifields with stronger effects on ipsilateral saccades.

To distinguish effects on visual and motor processing, Dominguez-Vargas et al. (2017) used a memory-guided saccade task, in which monkeys had to maintain fixation for a delay period after a target or targets were presented, and then make a saccade when a "go" cue was presented. Stimulation was applied for $200 \mathrm{~ms}$ in four time windows, $80 \mathrm{~ms}$ before or after presentation of the visual target or "go" cue. When stimulation was applied around target presentation during fixation, the only significant effect was to increase premature ipsilateral saccades, again contrasting with the effect of stimulation in the fixation task. As in the visually guided task, stimulation just before the "go" cue sped ipsilateral and 
slowed contralateral saccade onset, whereas stimulation after the "go" cue delayed all saccades. However, there was no effect on target choice.

Whereas results from previous pharmacological studies implied that the pulvinar may control "contralateral drive," this study suggests that the pulvinar plays a more nuanced role. Stimulation after the "go" cue delayed even contralateral saccades and stimulation before the "go" cue produced an ipsilateral bias, in contrast to pharmacological results. Additionally, when visual processing and motor execution were temporally dissociated, the choice bias observed in the visually guided task could not be reproduced by perturbing either process alone. This may indicate that behavioral effects of pulvinar stimulation depend either on the concurrence of visual processing and motor planning, or on the perturbation of both processes independent of their temporal relationship.

One technical concern with any experiment using electrical stimulation is that effects may be influenced by current spillover into neighboring regions. The brachium of the superior colliculus, a bundle of fibers to and from the superior colliculus, runs along the edge of the targeted region and might have been activated by pulvinar stimulation. This is of particular concern as past studies have found that effects that initially appeared to be due to pulvinar lesions indeed depended only on the fibers of passage in the brachium (Bender, 1988). Even at low currents, stimulation of the superior colliculus evokes contralateral saccades (Schlag et al., 1998). Lesions of the brachium cause contralateral slowing and an ipsilateral fixation offset (Sprague and Meikle, 1965; van Koningsbruggen et al., 2017). These results suggest that stimulation of the brachium could produce effects like those seen in the current study. However, Dominguez-Vargas et al. (2017) used magnetic resonance imaging guidance to keep the electrode within pulvinar, and did not stimulate within $1 \mathrm{~mm}$ of the brachium, ensuring that the primary site of stimulation was always the pulvinar itself.

Dominguez-Vargas et al. (2017) suggest several possible interpretations for the time-dependent effects of electrical stimulation, including that stimulation might have affected a mixed population of contralateral saccade-related neurons and fixation-related or hold neurons. Stimulation during fixation would offset the monkey's gaze contralaterally, as indicated by the increase in contralateral fixa- tion breaks. To maintain fixation, the monkey must compensate for this. If this compensation were to outlast the stimulation, it would potentiate ipsilateral responses, making them faster and more prevalent in the choice task. When stimulation occurs closer to saccade onset, on the other hand, the monkey would not have an opportunity to compensate before being prompted to respond, and the stimulation of contralateral-preferring neurons would favor contralateral responses, making them faster and more prevalent than ipsilateral responses. If stimulation also activated a population of fixation neurons, the initiation of any saccade would be delayed relative to the unstimulated case; and because of the contralateral drive described above, delays would be greater on ipsilateral saccades, as observed by Dominguez-Vargas et al. (2017).

Another related explanation for the time-dependent effects of electrical stimulation is that the pulvinar may carry anticipatory signals, as previously observed in a possible homologous region in the rat (Komura et al., 2001). In the memoryguided task of the current study, the firing rates of a subpopulation of pulvinar neurons increase after the presentation of a contralateral target, whereas they decrease for ipsilateral targets. During the following delay period, these deviations decay, returning to their common baseline at the time when the "go" cue is expected. This activity could represent a hold signal, which inhibits movement and decreases in anticipation of the saccade. Thus, if stimulation is applied early during an ipsilateral trial, firing rates are increased toward baseline, facilitating ipsilateral responses as observed. In contralateral trials, on the other hand, early stimulation would push firing rates further away from baseline, delaying the return to baseline and slowing responses. In contrast, if stimulation is applied later during either trial type, activity would normally be close to baseline and stimulation would push firing beyond target rates, resulting in the observed response delays.

The latter explanation is not unlike the one offered by Dominguez-Vargas et al. (2017). Both suggest that, as well as representing contralateral targets, some pulvinar neurons provide a hold signal, whether by maintaining fixation directly or preventing premature saccades. On a circuit level, this could be implemented through competition between pulvinar populations that represent current and potential future gaze positions. The effects of stimulation could be explained if this competition needed to be reconciled before saccades can be initiated. In an only weakly retinotopic region like the dorsal pulvinar, electrical stimulation activates neurons with a variety of spatial preferences. This activity would interact competitively with the activity representing the planned saccade, delaying responses compared with the unstimulated case. If conflicts represented within the same neural structure were more quickly resolved, such local interactions could also explain why stimulation effects on reaction time were weaker for contralateral saccades, when saccade preparation and electrical stimulation occurred in the same hemisphere. The offset of stimulation would remove the source of conflict between target- and stimulation-induced neural activity; and indeed, a significant proportion of saccades, especially ipsilateral ones, was delayed until after the offset of stimulation.

Overall, Dominguez-Vargas et al. (2017) showed that the influence of electrical stimulation on the dorsal pulvinar was highly time-dependent, resulting in opposite effects on target choice and response time, depending on whether stimulation was applied during fixation or close to saccade initiation. Delays in both ipsilateral and contralateral saccades induced by stimulation around saccade initiation indicate that stimulation does not merely act as "added contralateral drive," expanding on the results of previous pharmacological studies. The effects of stimulation rather suggest that pulvinar dynamics play a role in providing a competitive control mechanism for maintaining and shifting gaze.

\section{References}

Bender DB (1988) Electrophysiological and behavioral experiments on the primate pulvinar. Prog Brain Res 75:55-65. CrossRef Medline

Benevento LA, Standage GP (1983) The organization of projections of the retinorecipient and nonretinorecipient nuclei of the pretectal complex and layers of the superior colliculus to the lateral pulvinar and medial pulvinar in the macaque monkey. J Comp Neurol 217: 307-336. CrossRef Medline

Buschman TJ, Kastner S (2015) From behavior to neural dynamics: an integrated theory of attention. Neuron 88:127-144. CrossRef Medline

Dominguez-Vargas AU, Schneider L, Wilke M, Kagan I (2017) Electrical microstimulation of the pulvinar biases saccade choices and reaction times in a time-dependent manner. J Neurosci 37:2234-2257. CrossRef Medline

Gutierrez C, Cola MG, Seltzer B, Cusick C (2000) Neurochemical and connectional organiza- 
tion of the dorsal pulvinar complex in monkeys. J Comp Neurol 419:61-86. CrossRef Medline

Hardy SG, Lynch JC (1992) The spatial distribution of pulvinar neurons that project to two subregions of the inferior parietal lobule in the macaque. Cereb Cortex 2:217-230. CrossRef Medline

Komura Y, Tamura R, Uwano T, Nishijo H, Kaga K, Ono T (2001) Retrospective and prospective coding for predicted reward in the sensory thalamus. Nature 412:546-549. CrossRef Medline

Ma TP, Lynch JC, Donahoe DK, Attallah H, Rafols JA (1998) Organization of the medial pulvinar nucleus in the macaque. Anat Rec 250: 220-237. CrossRef Medline

Olshausen BA, Anderson CH, Van Essen DC
(1993) A neurobiological model of visual attention and invariant pattern recognition based on dynamic routing of information. J Neurosci 13:4700-4719. Medline

Petersen SE, Robinson DL, Keys W (1985) Pulvinar nuclei of the behaving rhesus monkey: visual responses and their modulation. J Neurophysiol 54:867-886. Medline

Petersen SE, Robinson DL, Morris JD (1987) Contributions of the pulvinar to visual spatial attention. Neuropsychologia 25:97105. CrossRef Medline

Schlag J, Pouget A, Sadeghpour S, Schlag-Rey M (1998) Interactions between natural and electrically evoked saccades: III. Is the nonstationarity the result of an integrator not instantaneously reset? J Neurophysiol 79:903-910. Medline
Sprague JM, Meikle TH Jr (1965) The role of the superior colliculus in visually guided behavior. Exp Neurol 11:115-146. CrossRef Medline

van Koningsbruggen $\mathrm{M}$, Koller $\mathrm{K}$, Rafal RD (2017) Deafferentation of the superior colliculus abolishes spatial summation of redundant visual signals. Front Syst Neurosci 11:9. CrossRef Medline

Wilke M, Turchi J, Smith K, Mishkin M, Leopold DA (2010) Pulvinar inactivation disrupts selection of movement plans. J Neurosci 30: 8650-8659. CrossRef Medline

Wilke M, Kagan I, Andersen RA (2013) Effects of pulvinar inactivation on spatial decisionmaking between equal and asymmetric reward options. J Cogn Neurosci 25:1270-1283. CrossRef Medline 